\title{
O MODELO BIOECOLÓGICO: DESVENDANDO CONTRIBUIÇÕES PARA A PRÁXIS DA ENFERMAGEM DIANTE DA VIOLÊNCIA DOMÉSTICA
}

\author{
Bioecological model: unveiling contributions to the nursing practice within domestic \\ violence \\ El modelo bioecológico: contribuciones a la divulgación de la praxis de enfermería \\ relacionados con la violencia doméstica
}

Maria Helena Lenardt ${ }^{3}$

\section{RESUMO}

Trata-se de um artigo de reflexão que teve o objetivo de discutir sobre os elementos do Modelo Bioecológico do Desenvolvimento Humano de Bronfenbrenner com a expectativa de encontrar contribuições para o conhecimento de enfermagem e sua práxis na interface com a violência doméstica. Compreender a complexidade do ser humano na perspectiva bioecológica possibilita 0 desenvolvimento de uma práxis transformadora no contexto da atuação da enfermeira diante da demanda de cuidados à pessoa que vivencia este tipo de violência. Considera-se que esta teoria sobre o desenvolvimento humano traz em sua estrutura elementos constituintes das inter-relações da pessoa e o ambiente ao seu entorno, como fatores determinantes na formação do indivíduo. Assim, ao se apropriar deste modelo, a enfermagem amplia o seu olhar sobre a multidimensionalidade humana, o que pode refletir no cuidado perante as situações de violência doméstica.

Palavras-chave: Enfermagem em saúde comunitária. Violência doméstica. Desenvolvimento humano.

\begin{abstract}
It is a reflection paper which objectified to discuss the elements of Bronfenbrenner's Bioecological Model on Human Development expecting to contribute for nursing knowledge and its praxis on domestic violence interface. Grasping human beings' complexity in the bioecological perspective enables the development of a transforming praxis in the context of nursing performance facing caring demand to a person who experienced this kind of abuse. It is considered that this theory on human development entails in its structure constitutive elements of people's interrelationships and their surrounding environment as the determining factors to shape individuals. Thus, by taking this model, nursing broadens its look on human multidimensionality which may be reflected in its praxis and care towards situations of domestic violence.
\end{abstract}

Keywords: Community health nursing. Domestic violence. Human development.

\section{Resumen}

Este es un artículo de reflexión que tuvo el propósito de discutir los elementos del modelo Bioecológico de Desarrollo Humano Bronfenbrenner, esperando encontrar las contribuciones al conocimiento y práctica de enfermería en su interface con la violencia doméstica. Comprender la complejidad de los seres humanos en la perspectiva bioecológica permite el desarrollo de una praxis transformadora en el marco de las acciones del enfermo frente a la demanda de atención a la persona que experimenta este tipo de violencia. Se considera que esta teoría sobre el desarrollo humano trae en su estructura elementos de relaciones interpersonales y el ambiente con su entorno, tales como factores determinantes en la formación del individuo. Así, la enfermería al apropiarse de este modelo amplía su mirada en el ser humano multidimensional, que puede reflexionar sobre su práctica frente a las situaciones de violencia doméstica.

Palabras claves: Enfermería en Salud Comunitaria. Violencia Doméstica. Desarrollo Humano

\footnotetext{
${ }^{1}$ Enfermeira. Mestranda em Enfermagem pelo Programa de Pós-Graduação em Enfermagem da Universidade Federal do Paraná (UFPR). Membro do Grupo de Estudos Multiprofissional em Saúde do Adulto (GEMSA) da UFPR. Curitiba-PR. Brasil. Email: janetebts@gmail.com; ²Enfermeira. Mestranda em Enfermagem pelo Programa de Pós-Graduação em Enfermagem da UFPR. Membro do GEMSA da UFPR. Curitiba-PR. Brasil. Email: tatiherreira@hotmail.com; ${ }^{3}$ Enfermeira. Doutora em Filosofia da Enfermagem. Professora Sênior do Programa de Pós-Graduação em Enfermagem da UFPR. Líder do Grupo Multiprofissional de Pesquisa sobre Idosos (GMPI) da UFPR. Curitiba-PR. Brasil. Email: Curitiba.helena@gmail.com; ㅌnfermeira. Doutora em Enfermagem. Professora do Programa de Pós-Graduação em Enfermagem da UFPR. Coordenadora do Curso de Graduação de Enfermagem. Membro do Grupo de Estudo da Família, Saúde e desenvolvimento Infantil GEFASED) da UFPR. Curitiba-PR. Brasil. Email: mazzas@ufpr.br; ${ }^{5}$ Enfermeira. Doutora em Enfermagem. Professora Associada do Departamento de Enfermagem e do Programa de Pós-Graduação em Enfermagem da Universidade Federal do Paraná. Líder do GEMSA da UFPR. Curitiba-PR. Brasil. Email: lililabronici@yahoo.com.br
} 


\section{INTRODUÇÃO}

A violência é um fenômeno que atinge a todos sem distinção de raça, cor, idade, etnia, gênero, condição social, profissão ou religião, traz em sua gênese uma multiplicidade de fatores ao envolver aspectos culturais, sociais, econômicos e políticos. Suas consequências são consideráveis para a totalidade humana, pois, ao afetar o indivíduo, atinge diretamente aqueles que compõem as suas relações sociais, como a família e a sociedade ${ }^{1}$.

A complexidade que envolve a violência doméstica acabou por colocá-la em posição de destaque nas agendas políticas a partir dos anos $80^{1}$ ao exigir que 0 agravo fosse enfrentado com propostas políticas no sentido de coibi-la e evitá-la, o que levou a organização de redes de serviços de referência para atendimento às pessoas vítimas de violência, bem como a organização de sistema de registros de informações sobre a violência doméstica, como forma de explicitar a magnitude do problema principalmente entre a população feminina, crianças e adolescentes.

Com o intuito de manter o foco da discussão desta reflexão, delimitamos a atenção na violência doméstica. Esta representa a agressão instalada no seio familiar, e se expressa nas formas sexual, física ou verbal, entre outras praticadas no âmbito da residência, envolve as relações proximais dos membros familiares, com laços ou não de consanguinidade e atinge tanto homens quanto mulheres, podendo resultar em danos à saúde; configura-se, assim, como um problema grave de saúde pública².

Os serviços de saúde constituem o lócus privilegiado e estratégico para detectar condições de risco e identificar as possíveis vítimas de violência doméstica. Além disso, estas ações representam possibilidades para identificar alguns dos elementos a serem avaliados pelo enfermeiro ao atender estas pessoas, e desta forma contribuir para explicitar o problema e dar a visibilidade compatível com sua magnitude e amplitude social.

De acordo com os dados disponibilizados pelo sistema de Vigilância de Violências e Acidentes (VIVA) do Ministério da Saúde, em 2008 foram notificados 8.766 casos de violência. Destes, $71 \%$ ocorreram entre as mulheres. As solteiras representaram $39 \%$ e as casadas ou com união estável, $23 \%$. Quanto ao local de ocorrência, a residência foi a mais predominante, com aproximadamente $57 \%$ dos casos, e a escola representou $13 \%$. A violência de repetição apresentou uma variação de $26 \%$ entre os homens a $40 \%$ entre as mulheres; nestes casos o evento violento já havia sido praticado anteriormente ${ }^{3}$.

Este sistema revelou ainda que, das 8.766 notificações, as maiores proporções (27\%) dos casos de violência notificados envolveram adolescentes e jovens entre 10 e 19 anos, seguidos de crianças de zero a nove anos (23\%) e adultos jovens entre 20 e 29 anos (19\%). Em relação aos tipos de violência, destacaram-se a física (56\%), seguida da psicológica e ou moral (41\%) e a sexual (31\%). Na questão de gênero, a violência apresentou distinções, sendo que a física (65\%) e a negligência (19\%) foram predominantes entre o sexo masculino, enquanto nas mulheres, além da agressão física (52\%), a violência psicológica e ou moral (49\%) e sexual (39\%) foram as mais notificadas ${ }^{3}$.

Desse modo, os casos de violência contra a mulher (6.236) apresentaram-se superiores em relação ao sexo masculino (2.530), em uma proporção de 2,4 para1.

Diante da violência doméstica, para propor um cuidado que se constitui em ações transformadoras, fazem-se necessários a compreensão do desenvolvimento humano como forma concreta de existir, um olhar do enfermeiro capaz de perceber 0 outro na sua singularidade com suas experiências, percepções e significados, bem como a noção da complexa rede multifatorial que envolve este tipo de violência. Deste modo, na concepção da Teoria Bioecológica, o desenvolvimento humano corresponde às multidimensões da pessoa no contexto de mundo complexo em que vive, no qual a violência doméstica ocorre.

Urie Bronfenbrenner nasceu em 29 de abril de 1917 em Moscou. Formou-se em Psicologia e Música pela Universidade de Cornell. Ainda criança mudou-se com seus pais para os Estados Unidos, onde morava em uma instituição rural para o tratamento de pessoas com retardo mental e acentuou o seu contato com diferentes modos de viver e pensar, experiências que fundamentaram a formulação e desenvolvimento dos pressupostos da Abordagem Bioecológica4.

A apropriação da Teoria Bioecológica amplia os conhecimentos de enfermagem na compreensão abrangente do indivíduo para o qual o cuidado será prestado, abre possibilidade de olhar o processo do desenvolvimento humano, diretamente associado às experiências de vida da pessoa, que influencia e é influenciada, de maneira contextualizada, interativa e processual, pelas suas interações ambientais.

Entende-se o ambiente natural, na visão Bioecológica, como aquele no qual objetos e atividades da vida cotidiana do sujeito acontecem e, por desenvolvimento humano, como um conjunto de transformações duradouras na vida da pessoa, na medida em que the causa impressões a partir da sua subjetividade construída na sua relação com o ambiente. 0 ser humano é visto como uma entidade em crescimento dinâmico e que gradativamente penetra no meio em que reside e 0 transforma na medida em que é transformado ${ }^{5}$.

É no ambiente que se estabelece o vínculo entre 0 desenvolvimento humano, e a violência doméstica, para essa reflexão, irá se restringir ao meio exterior, ao entorno mais próximo à pessoa, ou microssistema, como aborda a Teoria Bioecológica e se configura como um dos fatores constituintes do indivíduo no mundo, compreendido em um sistema com interações complexas. 
Considerar a pessoa em sua complexidade e sua forma de coexistir com a violência doméstica é pesar as implicações deste agravo na saúde do indivíduo que reflete no grupo familiar, e a Teoria Bioecológica emerge como possibilidade de compreensão do desenvolvimento humano a partir das suas relações com o meio no qual se insere. Deve-se considerar o sujeito em construção, com sua subjetividade diante da vida, e o saber da enfermagem sobre estes fatos contribui para um cuidado que propicia a emancipação dos envolvidos no contexto.

Orientados na Teoria Bioecológica de Bronfenbrenner, os estudos que têm como foco a família ${ }^{5-6}$ buscaram compreender a sua dinâmica relacional e identificaram que esta pode favorecer a negação da violência perpetrada, assim como promover fatores de proteção ${ }^{5}$. Outro estudo ${ }^{6}$ envolvendo a mesma temática constatou que as práticas educativas no meio familiar fortalecem os vínculos entre a criança e os demais membros do grupo e, assim, podem contribuir para o seu desenvolvimento. Ambos os estudos corroboram a ideia de que esta Teoria possibilita a análise da pessoa, família e seu contexto, bem como dos processos interativos influenciáveis no desenvolvimento humano.

Diante destas considerações, pretende-se refletir sobre os elementos do Modelo Bioecológico do Desenvolvimento Humano de Bronfenbrenner com o intuito de contribuir na construção do conhecimento de enfermagem e sua práxis na interface com a violência doméstica.

\section{O DESENVOLVIMENTO HUMANO NA PERSPECTIVA DO MODELO BIOECOLÓGICO DE BRONFENBRENNER}

Desde a segunda metade do século XX, com 0 surgimento das ciências que tratam das dimensões humanas, como as ciências sociais, a psicologia e as biocomportamentais, os estudos sobre o desenvolvimento humano buscam uma abordagem do curso da vida como processo caracterizado por inter-relações dinâmicas entre a pessoa e o ambiente, com influências mútuas ${ }^{7}$.

Compreender o indivíduo e suas inter-relações com o contexto no qual desenvolve a sua vida cotidiana têm sido o objetivo de pesquisas empíricas a partir das publicações de Urie Bronfenbrenner na década de 1970; entre elas está a Teoria Bioecológica do Desenvolvimento Humano. Segundo esta teoria, a pessoa se desenvolve por meio de processos de interações recíprocas e ativas com o seu meio imediato, no qual estão pessoas, objetos e símbolos ${ }^{8}$.

A teoria considera as constâncias e mudanças na vida do ser humano no decorrer de sua existência, os papéis e atividades cotidianas da pessoa, e suas implicações no desenvolvimento intelectual, emocional, social e moral, além do meio ambiente no qual o indivíduo está inserido, bem como as mudanças sofridas nos eventos no decorrer dos tempos na sua história 5 .
Destarte, há reciprocidade entre as relações humanas e o ambiente, no processo de construção ao longo da existência do indivíduo, o qual imprime suas impressões no mundo ao seu entorno e como este interfere no eu. Na perspectiva da Teoria Bioecológica, o desenvolvimento humano é compreendido como um fenômeno complexo que ocorre ao nível de quatro núcleos principais: as interações proximais que promove 0 desenvolvimento; as características pessoais; 0 contexto e 0 tempo ${ }^{4}$.

A teoria em pauta trata da interação que a pessoa estabelece com seus pares, objetos e símbolos em diferentes contextos ecológicos através do tempo, conduzindo o desenvolvimento humano a uma conformação segundo as características biopsicológicas da pessoa ao longo da vida 90 desenvolvimento humano se caracteriza pela acumulação de aquisições e conhecimentos a partir das experiências vividas, e a forma como são conduzidos influencia no desenvolvimento das potencialidades do ser humano.

Nesta perspectiva, o desenvolvimento humano, proposto por Bronfenbrenner é processual, interativo e bidirecional, em um movimento dinâmico ${ }^{10}$ no qual ocorre 0 intercâmbio entre as peculiaridades do indivíduo e do ambiente de modo a produzir mudança nas características da pessoa no curso de sua existência.

A teoria considera o desenvolvimento humano um entrelaçar de processos psicológicos com as multideterminações ambientais, resultando em domínios evolutivos de aquisições das competências e habilidades pessoais, para conduzir e direcionar seu próprio comportamento, bem como lidar com as dificuldades nas mais diversas situações ${ }^{9}$.

Trata-se de um paradigma emergente que aborda a complexidade e a racionalidade plural, orientada em concepções das questões humanas, como a subjetividade, valorização da ética e da ecologia, e se configura como movimento de julgar a realidade que se opõe à racionalidade, ao reducionismo, ao mecanicismo, ao cientificismo orientado no positivismo, no qual sujeito e objeto não se relacionavam ${ }^{11}$.

As dinâmicas do desenvolvimento humano dentro das estruturas propostas por Bronfenbrenner ocorrem como um processo interativo entre o indivíduo e o contexto através do tempo, têm como foco principal as interações ${ }^{9-10}$, nas quais 0 ser humano se apresenta como agente ativo do seu desenvolvimento, capaz de transformar o ambiente e transformar-se.

Como proposição central, a Teoria Bioecológica traz a visão do desenvolvimento humano como um processo de interação envolvendo organismo-ambiente; este último é concebido como um conjunto de estruturas denominadas micro, meso, exo e macrossistema, representados pelos ambientes nos quais o indivíduo se insere, como a casa, a escola, a igreja, os serviços de saúde e o Estado, respectivamente. Contudo, o 
nível mais profundo do desenvolvimento do indivíduo refere-se ao microssistema ${ }^{4.9}$.

Importa destacar o microssistema, tanto pela sua relevância no conjunto das relações mais imediatas do indivíduo quanto pela qualidade destas, como lócus relevante para a atenção do enfermeiro no sentido de atuar em conjunto com os demais membros do convívio da pessoa em situação de violência doméstica a fim de estabelecer propostas de cuidado de forma articulada, pactuada e passível de transformação de uma realidade violenta para outra que realmente promova as pessoas para um viver melhor.

É no microssistema que o indivíduo desenvolve um conjunto de papéis sociais primários com apreensão dos valores padronizados pela sociedade como base para o seu desenvolvimento. 0 mesossistema envolve a participação ativa da pessoa e a interação com outros ambientes, o que denota ser um sistema composto de microssistemas. Já o exossistema constitui-se por ambientes fora do convívio familiar; contudo, a vivência da pessoa influencia os demais membros do grupo e o macrossistema se configura como o mais amplo dos sistemas e engloba padrões de organização social, cultural e ideológica ${ }^{5}$.

A partir da teoria ora apresentada, no sentido de estabelecer relação entre a violência doméstica e microssistema como potencial de atuação para a enfermeira, pode-se dizer que o grupo familiar como espaço das relações primárias do indivíduo representa uma unidade social e comportamental interdependente entre os seus membros, ou seja, a forma de agir de um interfere no comportamento do outro, o que remete a análise de que a conduta violenta de um para com outro poderá ter repercussões significativas nas vidas de ambos.

\section{O MODELO BIOECOLÓGICO DE BRONFENBRENNER: CONTRIBUIÇÕES PARA A PRÁXIS DA ENFERMAGEM PERANTE AS SITUAÇÕES DE VIOLÊNCIA DOMÉSTICA}

A práxis pode ser definida como toda atividade humana que se expressa no trabalho com vistas a um fim. Como expressão da atitude do sujeito diante da realidade, exige uma atividade cognitiva que mobiliza o indivíduo a transformar o seu mundo e ser transformado ${ }^{11}$. Neste sentido, o cuidador e o ser cuidado experimentam as mudanças concretas, o primeiro por perceber o resultado do seu trabalho e conhecimento e 0 segundo por sentir-se contemplado nos aspectos elementares do seu desenvolvimento que caracteriza a sua existência.

Pensar a enfermagem como práxis diante das situações de violência doméstica é refletir sobre as necessidades do ser humano que vivencia ou vivenciou esse evento traumático, e como se expressa o cuidado para esse sujeito que traz consigo a complexidade tanto do fenômeno quanto do ser humano.

Compreende-se, ainda, práxis como "teoria em ação, prática em movimento", envolve participação para alterar uma realidade com esforço e interesse coletivo ${ }^{12: 179}$. Com o estabelecimento do cuidado compartilhado com a pessoa em situação de violência doméstica, surge a possibilidade de troca mútua e a construção de uma rede de relações propícias para promover o ambiente familiar mais saudável, que promova o desenvolvimento saudável dos indivíduos, o que reflete a amplitude do olhar da enfermeira sobre a pessoa.

A violência doméstica apresenta-se como demanda à prática de enfermagem, no âmbito familiar que envolve os seus integrantes com laços de consanguinidade ou conviventes. Esse tipo de violência gera um ambiente de insegurança às suas vítimas que geralmente se encontram em posição de desigualdade hierárquica, autoritária e de poder ${ }^{5}$.

Ao pensar o cuidado às vítimas de violência doméstica, a enfermagem deve considerar os fatores relacionados com a situação, olhar além do fato ou queixa apresentada, pensar globalmente sobre os fatores que contribuem para a produção da violência e direcionar as suas aç̃̃es de cuidado à pessoa considerando as suas especificidades e singularidades.

Neste sentido, a Teoria Bioecológica proposta por Bronfenbrenner apresenta aspectos importantes sobre 0 desenvolvimento humano, que contribuem para que a enfermagem desenvolva a visão ampliada do indivíduo. Deste modo, responde de forma efetiva diante das suas demandas, de maneira mais específica àquelas relacionadas à violência doméstica, na perspectiva do olhar, que possibilita o cuidado contextualizado, que considere 0 indivíduo nas suas multidimensões, a sua subjetividade, com vistas a motivá-lo para a ação transformadora da realidade que o circunda como sujeito ativo e reflexivo.

Ao aplicar a Teoria Bioecológica no nível micro, diretamente no núcleo familiar, o cuidado de enfermagem deve alcançar a dimensão individual e coletiva, contemplando a família com ações que promovam a autoestima dos integrantes do grupo e favoreçam a capacidade de compreensão dos problemas, escolhas de alternativas para a resolução dos conflitos e mobilização de recursos internos e externos, de maneira que as relações positivas estabelecidas no microssistema possam repercutir em outros ambientes que compõem o entorno da pessoa.

0 cuidado de enfermagem com enfoque na pessoa deve considerar a sua necessidade e multidimensionalidade; destarte o Modelo Bioecológico de Urie Bronfenbrenner emerge como possibilidade de oferecer subsídios para conhecimento sobre essas dimensões que permeiam o desenvolvimento humano, ao apresentar elementos constitutivos da pessoa, em um processo dinâmico, no qual ocorre a interação entre o indivíduo e o ambiente em que está inserido.

A violência doméstica ganha mais visibilidade e dimensões contextuais quando analisada sob a perspectiva da teoria em questão, pois assim se possibilita à enfermeira cuidar de forma articulada com outros serviços e profissionais, permite a coparticipação dos envolvidos no problema, desprovidos de conceitos pré-concebidos sobre o indivíduo, e garante, dessa forma, um cuidado com possíveis resultados mais concretos. 
Ao pensar o cuidado de enfermagem no contexto da violência doméstica busca-se uma atuação centrada no indivíduo, considerando o meio no qual se insere, de forma mais específica, no microssistema, que se configura no espaço familiar, no qual ocorrem relações sociais proximais. Com esta constatação, a Teoria Bioecológica se apresenta como subsídio para orientar um cuidado que promova a saúde das pessoas envolvidas, com vistas à transformação da sua realidade e manutenção de um desenvolvimento de vida saudável.

A atenção à saúde na perspectiva do Modelo Bioecológico vai ao encontro do novo paradigma de atenção à saúde, porquanto coaduna uma fonte de conhecimento sobre a dimensionalidade humana como subsídios para o profissional ampliar o seu olhar para além do corpo biológico, e a teoria proposta por Bronfenbrenner propicia considerar o indivíduo e sua subjetividade na forma de se relacionar com o mundo.

Nesse sentido, o cuidado de enfermagem deve ir para além da execução de procedimentos em uma lógica que pensa a pessoa como a soma das suas partes de forma simplificada; no novo paradigma, faz-se necessária a compreensão abrangente e profunda do ser, que contemple a sua integralidade e a sua subjetividade e as diversidades dos contextos nos quais 0 indivíduo se insere. Assim, o profissional de enfermagem busca desenvolver o cuidado que satisfaça às necessidades da pessoa, considerando-a como um todo, com ênfase nas suas especificidades ${ }^{13}$. É um cuidado sob demanda, e não prescritivo e diretivo.

As necessidades humanas emergidas na atual conjuntura de mundo impulsionam a enfermagem para uma nova tendência de reformular competências e atitudes profissionais, na direção de um saber/fazer com capacidade de compreensão e análise, cuidando do indivíduo em suas dimensões, bem como definindo 0 ser da enfermagem perante a sociedade ${ }^{13}$.

A partir da articulação entre o saber e o fazer espera-se um cuidado transformador que contemple as multidimensões do indivíduo, oferecendo-lhe condições para que consiga encontrar um caminho de transformação ou alteração da sua condição humana, e, nesta prática reflexiva, a ação de enfermagem deve estar pautada em competências e habilidades alicerçadas nos diversos tipos de conhecimentos, com vistas a promover o viver saudável, atender às necessidades e agravos à saúde, bem como cuidar das situações de cronicidade, no decorrer da existência humana ${ }^{14}$

É importante salientar que a partir do conhecimento científico e reflexivo, a enfermeira vislumbra a possibilidade de ampliar o horizonte sobre o campo do cuidado e ao mesmo tempo focalizar a sua direção, considerando as suas minúcias. Para tanto, é necessário se apropriar de outras áreas dos saberes a exemplo das ciências sociais, com vistas a firmar o olhar "holográfico das condições socioculturais que se estabelecem entre sujeitos em suas vivências"14:561.

Ao fazer analogia à prática da enfermagem, o olhar, na concepção filosófica, é a capacidade de captarmos o mundo à nossa volta com sabedoria; diz-se que o outro será captado com profundidade, em sua complexa individualidade e singularidade. Exige-se operações do pensamento, com efeito, "onde aparece o olhar, a visão se descompõe"15:51.

Sob os aspectos apresentados, o profissional enfermeiro tem a possibilidade de apreender a subjetividade que envolve o indivíduo e o seu processo de vida, considerando suas experiências, sua subjetividade e sua história, ademais implica gerar condições para saúde/doença, bem como para o seu desenvolvimento.

A partir desta constatação, a Teoria Bioecológica de Bronfenbrenner emerge como possibilidade de conhecimento da enfermeira no sentido de oferecer um cuidado, fundamentado e contextualizado, tanto para a pessoa que experiencia a violência doméstica como para a família haja vista que, segundo a teoria, ambos estão imersos no microssistema com relações recíprocas que imprimem em suas existências as marcas deste tipo de violência. Especificamente, deve-se assistir com eficácia ou, dito de outra forma, com uma práxis que vise mobilizar os envolvidos para transformar a própria realidade.

Apropriar-se da Teoria Bioecológica do Desenvolvimento Humano de Urie Bronfenbrenner, com o propósito de conhecimento, significa qualificar o cuidado de Enfermagem, pela amplitude do olhar sobre a pessoa. É vislumbrar respostas na recuperação de pessoas vítimas de violência doméstica em um determinado contexto, que reflete no desenvolvimento humano, afetando as condições de vida do indivíduo.

\section{CONSIDERAÇÕES FINAIS}

A violência gerada no âmbito doméstico traz em suas origens processos multicausais e, ao se relacionar com a distribuição desigual de autoridade e poder entre os membros da família, expõe seus integrantes a experiências que deixam marcas visíveis e invisíveis no desenvolvimento humano.

0 problema da violência doméstica é uma realidade nos serviços de atenção primaria à saúde e, nesse contexto, a enfermagem deve estar preparada para atender estas necessidades humanas emergidas com competências e atitudes profissionais, na direção de um saber/fazer com capacidade de compreensão e análise. Além disso, oferece um cuidado que atenda necessidades do indivíduo e contemple as suas múltiplas dimensões.

Sendo assim, o processo de desenvolvimento humano expressa-se nas experiências do indivíduo, e compreendê-lo na perspectiva bioecológica dá fulcro para a Enfermagem se aproximar das dimensões humanas e se instrumentalizar para planejar um cuidado que irá ao encontro das necessidades da pessoa, com uma práxis engajada e emancipatória, de maneira que o indivíduo possa se movimentar para mudar a sua condição humana no contexto da violência doméstica. 
Neste sentido, a atuação da enfermeira poderá ser mais efetiva na medida em que se propõe um cuidado pautado na corresponsabilidade entre os integrantes do âmbito familiar, bem como articulado aos demais serviços e profissionais. Contudo, o diferencial do cuidado de enfermagem, prestado por um profissional que tem o conhecimento e a reflexão sobre o desenvolvimento humano na perspectiva bioecológica, está no fato de disponibilizar conhecimento para um cuidado desprovido de conceitos pré-concebidos sobre a pessoa que vivencia cotidianamente a violência doméstica. Deste modo, 0 cuidado é um olhar atento à medida que se estabelecem os processos interacionais junto ao ambiente, pessoas, coisas e símbolos.

Na mudança paradigmática de olhar o indivíduo, o cuidado de enfermagem deve buscar a contextualização, no sentido de compreender a complexidade do problema e obter resultados que satisfaçam a necessidade apresentada. Assim, sendo cuidar da pessoa em situação de violência doméstica significa vê-la inserida em ambiente no qual estabelece interrelações, de maneira que suas atitudes interferem no contexto na medida em que são influenciadas por ele.

\section{REFERÊNCIAS}

1.Labronici LM, Ferraz, MIR, Trigueiro, TH, Fegadoli, D. Perfil da violência contra a mulheres atendidas na Pousada de Maria. Rev. Esc. Enferm. USP (Online). 2010 mar [citado 2011 Mar 09]; 44(1): [aprox. 8 telas]. Disponível em: <http://www.scielo.br/pdf/reeusp/v44n1/a18v44n1.pdf>.

2.Ministério da Saúde (BR). Enfrentando a Violência contra a Mulher. Brasilia(DF): Secretaria Especial de Políticas para as Mulheres; 2005.

3.Ministério da Saúde (BR). Viva: vigilância de violências e acidentes, 2008 e 2009. Brasilia(DF): Secretaria de Vigilância em Saúde, Departamento de Análise de Situação de Saúde; 2010.

4.Bronfenbrenner U, editor. Making human beings human: bioecological perspectives on human development. Thousand Oaks (EUA): Sage Publications; 2005.

5.Martins CS, Ferriani MGC, Silva MAI, Zahr NR, Arone KMB, Roques EMST. A dinâmica familiar na visão de pais e filhos envolvidos na violência doméstica contra crianças e adolescentes. Rev. Latino-Am. Enfermagem (Online). 2007 set/out [citado 2011 mar 09] 15(5): [aprox. 6 telas]. Disponível em:<http://www.scielo.br/ scielo.php?pid=S010411692007000500002\&script=sci_arttext\&thng=pt>.

6.Martins E, Szymanski H. A abordagem ecológica de Urie Bronfenbrenner em estudos com famílias. Estud. pesqui. psicol. [on line]. 2004 jun [citado 2011 mar 09] 2004(1). Disponível em: <http://pepsicbusaludorg/ scielo.php?script=sci_arttext\&pid=S180842812004000100006>.
7.Silva SC, Dessen MA, Costa AL Jr. As contribuições da ciência do desenvolvimento para a psicologia da saúde. Ciênc. saúde coletiva. 2011; 16 (sup.1): 1599-609.

8.Böing E, Crepaldi MA, More CLO0. Pesquisa com famílias: aspectos teórico-metodológicos. Paidéia (Ribeirão Preto). 2008; 18(40): 251 66.

9.Cecconello AM, Koller SH. Inserção Ecológica na Comunidade: Uma Proposta Metodológica para o Estudo de Famílias em Situação de Risco. Psicol. reflex. crit. 2003; 16(3): 515-24.

10.Siqueira AC, Dell'Aglio DD. 0 impacto da institucionalização na infância e na adolescência: uma revisão de literatura. Psicol. soc. 2006 jan/abr; 18(1): 71-80.

11.Vázquez, S. A. Filosofia da práxis. São Paulo: Expressão Popular; 2007.

12.Vale EG, Pagliuca LMF, Quirinos RHR. Saberes e práxis em enfermagem. Esc Anna Nery. 2009 mar; 13(1): 174-80.

13.Azevedo DM, Costa LM, Almeida Júnior JJ, Enders BC, Menezes RMP. Paradigmas emergentes: um ensaio analítico. Rev. eletrônica enferm [on line]. 2008 [citado 2010 mai 06]; 10(3): [aprox.8 telas]; Disponível em $<$ http://www.fen.ufg.br/revista/v10/n3/v10n3a30.htm>.

14.Erdmann AL, Schlindwein BH, Sousa FGM. A produção do conhecimento: diálogo entre os diferentes saberes. Rev. bras. enferm. 2006 jul/ago; 59(4): 560-4.

15.Quinet A. Um olhar a mais: ver e ser visto na psicanálise. Rio de Janeiro: Jorge Zahar; 2004. 